OPEN ACCESS

Edited by:

Gregor Starc,

University of Ljubljana, Slovenia

Reviewed by:

Zhen-Bo Cao,

Shanghai University of Sport, China

Tao Huang,

Shanghai Institute of Nutrition and Health (CAS), China

${ }^{*}$ Correspondence:

Jun Ma

majunt@bjmu.edu.cn

Yi Song

songyi@bjmu.edu.cn

Specialty section

This article was submitted to

Children and Health,

a section of the journal

Frontiers in Public Health

Received: 12 April 2021

Accepted: 10 August 2021

Published: 03 September 2021

Citation:

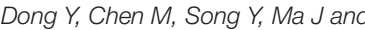

Lau PWC (2021) Geographical

Variation in Physical Fitness Among Chinese Children and Adolescents

From 2005 to 2014.

Front. Public Health 9:694070. doi: 10.3389/fpubh.2021.694070

\section{Geographical Variation in Physical Fitness Among Chinese Children and Adolescents From 2005 to 2014}

\author{
Yanhui Dong ${ }^{1}$, Manman Chen ${ }^{1}$, Yi Song ${ }^{1 *}$, Jun Ma ${ }^{1 *}$ and Patrick W. C. Lau ${ }^{2,3}$ \\ ${ }^{1}$ Institute of Child and Adolescent Health, School of Public Health, Peking University, Beijing, China, ${ }^{2}$ Department of Sport, \\ Physical Education and Health, Hong Kong Baptist University, Hong Kong, China, ${ }^{3}$ Laboratory of Exercise Science and \\ Health, BNU-HKBU United International College, Zhuhai, China
}

Introduction: To examine the geographical variation in change in the levels of physical fitness from 2005 to 2014 among Chinese children and adolescents.

Methods: A total of 663,813 children 7 to 18 years of age with physical fitness data in 2005, 2010 and 2014, participated in the study. Physical fitness was assessed using six components, and their standardized scores were aggregated to form a summary physical fitness indicator (PFI).

Results: Over the study period, there was an increase in the proportion of high PFI (from 15.3\% in 2005 to $19.0 \%$ in 2014, $P_{\text {trend }}<0.05$ ) and a decrease in the proportion of low PFI (from 30.6 to $29.8 \%, P_{\text {trend }}<0.05$ ). Children and adolescents in the eastern provinces of China showed a high proportion of high PFI with an increasing trend over time, while those in the western provinces had a high proportion of low PFI with a decreasing trend over time $(P<0.05)$.

Conclusions: The comprehensive physical fitness among Chinese children showed an improved trend from 2005 to 2014. Region-specific interventions with priority policies could be useful to sustainably narrow geographical variation in Chinese children, especially in the western provinces.

Keywords: physical fitness, geographical variation, children and adolescents, China, trend analysis

\section{INTRODUCTION}

Physical fitness is the body's ability to perform activities or motions effectively and efficiently. Usually, physical fitness is a comprehensive concept that combines various abilities together, such as muscular strength and endurance, cardiorespiratory endurance, and motor ability (1). Thus, a universal comprehensive physical fitness indicator (PFI) is used to define composite fitness among children and adults $(2,3)$. Previous studies have physical fitness was associated with the development of cardiovascular diseases (CVD) in both children and adults (4-6).

Physical fitness is considered to be a reflection of outcomes of physical activity (PA), so many physical exercise related factors were considered to affect physical fitness (7-9). Previous study found that the physical fitness among children and adolescents has been a world-wide decline since the late 1950s, especially in the maximal long-distance running performance of Asian children and adolescents, including China (10). One study using a national study in Chinese children and adolescents demonstrated that China has followed global trends in exhibiting a steadily deterioration in the levels of physical fitness, particularly in the domain of cardiorespiratory fitness (11-13). 
With the increasing number of factors that contribute to physical fitness deterioration among Chinese children and adolescents, such as the increased prevalence in obesity, lack of physical exercise, increased academic burden and use of electronic devices, more public health measures need to be focused on priority areas and regions. However, there has been a lack of systematic research in examining changes in geographic variations of comprehensive physical fitness using populationlevel prevalence data. Therefore, the objective of this study is to examine geographical variation in the levels of physical fitness among Chinese school-aged children and adolescents using three successive national cross-sectional surveys from 2005 to 2014.

\section{METHOD}

\section{Study Design and Participants}

Data were extracted from CNSSCH in 2005, 2010 and 2014. The CNSSCH was the largest nationally representative surveys comprising multiple core components of physical fitness among Chinese children and adolescents aged 7-18 years, and the $\mathrm{CNSSCH}$ was jointly launched by the Ministry of Education, the Ministry of Health, the Ministry of Science and Technology, the State of Nation Affairs, and the State Sports General Administration of People's Republic of China in order to investigate the constitution and health of children and adolescents. The details of the design of $\mathrm{CNSSCH}$ have been described in the previous studies (14). The CNSSCH used a multistage stratified cluster sampling design and included 31 Chinese provinces but did not cover Hong Kong, Macau and Taiwan. Initially, children and adolescents in each province, except Tibet, were stratified by three levels of prefecture cities (i.e., upper, moderate, low) based on their socioeconomic status. Then, in each city of three sub-provincial levels, children and adolescents were also stratified by urban and rural areas based on their residence location. Within these stratified areas since 1985, schools covering students aged 7-18 years including primary, middle and high schools were randomly selected, and the sampling procedure has remained uniform in each subsequent survey year. From these schools, all students in the randomly selected classes were included in the survey. Following inclusion and exclusion criteria and obtaining informed consent (as shown in previous studies) $(14,15)$, at least 50 Han students in each age group from 7-18 years of boys or girls in urban and rural areas were included in the survey. As participants in Tibet were minority students, only children in Lasa city were surveyed for feasibility reasons. At last, a total of 663,813 children and adolescents aged $7-18$ years $(234,289$ in $2005,215,223$ in 2010 , and 214,301 in 2014) were collected for the geographical variations trends analyses of physical fitness.

\section{Physical Fitness Measurements and Categories}

Participants in the survey underwent a complete physical fitness test according to the same protocol at all survey sites. All physical fitness tests were implemented in PE classes by special trained PE teachers who have passed the measurement examination. A school doctor was present to prevent children and adolescents from being injured during the physical fitness test, and a project supervisor was present to monitor whether the physical fitness test was carried out as required and to provide necessary guidance. Six physical fitness components (forced vital capacity (FVC), standing long jump (SLJ), sit-and-reach (SR), body muscle strength (BMS), 50 meter dash and endurance running) were included in our study and were measured by a team of trained technicians following a standardized procedure. FVC, SLJ, SR, and 50 meter dash were measured at each age from 718 years. According to the changing physical capabilities with age and sex, BMS was evaluated by oblique body pull-ups (boys aged 7-12 years), pull-ups (boys aged 13-18 years) and 1 min sit-ups (girls aged 7-18 years). Speed and speed endurance were evaluated by interval $50 \mathrm{~m}$ dash time and time for long distance running $(50 \mathrm{~m} \times 8$ shuttle run for both boys and girls aged 712 years; 1,000 $\mathrm{m}$ endurance running for boys aged $13-18$ years; and $800 \mathrm{~m}$ endurance running for girls aged 13-18 years). All the measuring instruments were consistent at each survey site and calibrated before use. All the students in the final analysis took each test simultaneously. Almost $100 \%$ of the students took all tests on the same day. The sample sizes and proportion of participants in each item test in each survey year are shown in the Supplementary Table 1. A total of 234,289, 215,223, and 214,301 chidlren and adolescents participated the physical fitness tests, and total valid participants for each physical fitness component tests in 2005 and 2010 exceeded $99.0 \%$ and even reached at 100\% in 2014.

Allowing for the different items in boys and girls of different age groups and difficulties of comparison, sex- and age-specific standardized values were calculated for each physical fitness component. Based on the reference population with median values and standard deviation (SD) of each physical fitness component that was defined in previous studies $(2,12)$. $Z$ scores of each component were calculated as an individuals' item value minus the median, divided by the SD for that child's age and sex in the reference population. PFI was calculated by summing the standard values for each of the six items: $P F I=$ standardized values of $F V C+S L J+S R+B M S+(-50 m$ dash $)+(-$ endurance running). Based on the percentile of the PFI in the reference population in 1985, we categorized the PFI into five levels: low level ( $<20$ th), low-middle level ( $\geq 20$ th and $<40$ th), middle level ( $\geq 40$ th and $<60$ th), middle-high level $(\geq 60$ th and $<80$ th), high level ( $\geq 80$ th).

\section{Statistical Analysis}

Descriptive statistics were used to describe the demographic characteristics of the study population in CNSSCH. Chi-square tests were used to examine between group differences in categorical variables. Stacking scales diagrams were employed to examine trends in the levels of physical fitness across the study period. To analyze geographical variation, we compared the proportion of different levels of physical fitness in each province (excluding Hong Kong, Macau, and Taiwan) across the three survey years. To examine geographical variation, the thirty-one mainland Chinese provinces were divided into four regions: East, Central, West, and Northeast, in accordance with the geographical standard division from the Chinese National 
TABLE 1 | The sample size and distribution of each physical fitness component from 2005 to 2014 in CNSSCH.

\begin{tabular}{|c|c|c|c|}
\hline Survey year & 2005 & 2010 & 2014 \\
\hline Total sample size, $\mathrm{n}$ & 234,289 & 215,223 & 214,301 \\
\hline \multicolumn{4}{|l|}{ Physical fitness measurement } \\
\hline Forced vital capacity, $n$ (\%) & 234,003 (99.88) & $215,171(99.98)$ & $214,301(100.00)$ \\
\hline Original values (Mean (SD), ml) & 2,017.1 (923.0) & 2,095.4 (947.1) & $2,210.0(1,001.8)$ \\
\hline Standardized values, Mean (SD) & $-0.88(1.27)$ & $-0.66(1.33)$ & $-0.41(1.41)$ \\
\hline Standing Long Jump, $n$ (\%) & 234,229 (99.97) & $215,001(99.90)$ & $214,301(100.00)$ \\
\hline Original values (Mean (SD), cm) & $165.9(37.0)$ & $166.4(37.5)$ & $163.5(38.1)$ \\
\hline Standardized values, Mean (SD) & $0.25(1.09)$ & $0.28(1.13)$ & $0.12(1.17)$ \\
\hline Sit and Reach, $n$ (\%) & 234,241 (99.98) & 214,983 (99.89) & $214,301(100.00)$ \\
\hline Original values (Mean (SD), cm) & $9.2(6.8)$ & $9.4(6.7)$ & $9.1(7.1)$ \\
\hline Standardized values, Mean (SD) & $0.14(1.19)$ & $0.19(1.17)$ & $0.14(1.23)$ \\
\hline Body Muscle Strength, $n(\%)$ & 234,086 (99.91) & 214,741 (99.78) & $214,301(100.00)$ \\
\hline \multicolumn{4}{|l|}{ Original values, Mean (SD): } \\
\hline Oblique Body Pull-ups (Boys aged 7-12 years) & $30.8(22.6)$ & $26.2(18.4)$ & $23.8(16.0)$ \\
\hline Pull-Ups (Boys aged 13-18 years) & $3.9(4.1)$ & $4.0(5.1)$ & $3.4(3.6)$ \\
\hline 1-min Sit-Ups (Girls aged 7-18 years) & $26.3(10.8)$ & $25.3(10.8)$ & $27.8(10.8)$ \\
\hline Standardized values, Mean (SD) & $0.39(1.33)$ & $0.27(1.24)$ & $0.32(1.22)$ \\
\hline 50 Meter Dash, $n(\%)$ & 234,164 (99.95) & 214,884 (99.84) & $214,301(100.00)$ \\
\hline Original values (Mean (SD), s) & $9.5(1.5)$ & $9.6(1.5)$ & $9.5(1.5)$ \\
\hline Standardized values, Mean (SD) & $-0.02(1.24)$ & $0.01(1.26)$ & $-0.07(1.28)$ \\
\hline Endurance running, $n(\%)$ & 233,301 (99.58) & 213,719 (99.30) & $214,301(100.00)$ \\
\hline \multicolumn{4}{|l|}{ Original values (Mean (SD), s) } \\
\hline $50 \mathrm{~m} \times 8$ shuttle run (Students aged $7-12$ years) & 126.7 (19.3) & $127.1(17.3)$ & $126.7(17.3)$ \\
\hline $1,000 \mathrm{~m}$ run (Boys aged 13-18 years) & $272.1(38.3)$ & $271.5(39.0)$ & $274.3(41.2)$ \\
\hline $800 \mathrm{~m}$ run (Girls aged 13-18 years) & $261.7(32.4)$ & $260.9(32.8)$ & $261.5(34.2)$ \\
\hline Standardized values, Mean (SD) & $1.00(1.52)$ & $1.03(1.56)$ & $1.02(1.55)$ \\
\hline
\end{tabular}

$n(\%)$, the sample size and percentage completing each physical fitness measurement.

Bureau of Statistics (NBS) (Supplementary Figure 1) (16). We used the Cochran-Armitage trend test to calculate the $P_{\text {trend }}$ values. Statistical analyses were conducted with Stata (version 15.0) statistical software. Two-sided $P<0.05$ was considered statistically significant.

\section{RESULTS}

\section{Study Population Characteristics}

The characteristics of the study population of $\mathrm{CNSSCH}$ are presented in Supplementary Table 1. The proportion of sample sizes for physical fitness from 2005 to 2014 and questionnaire survey in 2014 were balanced across sex, age, provinces, and urban and rural areas. The mean of original and standardized values of FVC got improvement, but other five physical fitness components among children and adolescents presented a fluctuating tends from 2005 to 2014 (Table 1).

\section{Trend in Physical Fitness}

Physical fitness, as indexed by PFI, improved with an increased proportion of children and adolescents who performed at above middle-high levels of PFI $\left(P_{\text {trend }}<0.05\right)$, and a decreased proportion of those who scored below low-middle levels of PFI $\left(P_{\text {trend }}<0.05\right)$. Boys and girls showed a similar increasing trend in the proportion of their physical fitness from 2005 to 2014 with both girls and boys showing a significant increase in the proportion of high levels of PFI (13.5\% in 2005 to $19.8 \%$ in 2014, $P_{\text {trend }}<0.05$ ) for girls, $17.1 \%$ in 2005 to $18.2 \%$ in 2014 , $P_{\text {trend }}<0.05$ for boys, respectively). The proportion of low levels of PFI decreased in girls, but increased in boys $(30.9-27.0 \%$ in girls, $P_{\text {trend }}<0.05$, and $30.4-32.6 \%$ in boys, $P_{\text {trend }}<0.05$, respectively). With respect to residence, results from urban and rural students revealed a similar trend, showing an increased proportion of those who performed above middle-high levels of PFI and a decreased proportion of those who performed below low-middle levels of PFI $\left(P_{\text {trend }}<0.05\right)$ (Figure 1). Among the four age groups, the proportion of the high levels of PFI showed an increased trend from 2005 to 2014, particular for those aged 13-15 years from 13.4 to $22.1 \%$ during the 10 years $\left(P_{\text {trend }}<\right.$ $0.05)$. Also, children and adolescents aged 13-15 years witnessed the largest decrease for low levels of PFI from 33.3\% in 2005 to $28.5 \%$ in $2014\left(P_{\text {trend }}<0.05\right)$ (Figure 2).

\section{Geographical variations}

With regard to the level of PFI (data shown in Figure 3), over the study period, children living in eastern Chinese provinces showed a relatively higher proportion of physical fitness compared to those living in western provinces. For example, children in the 


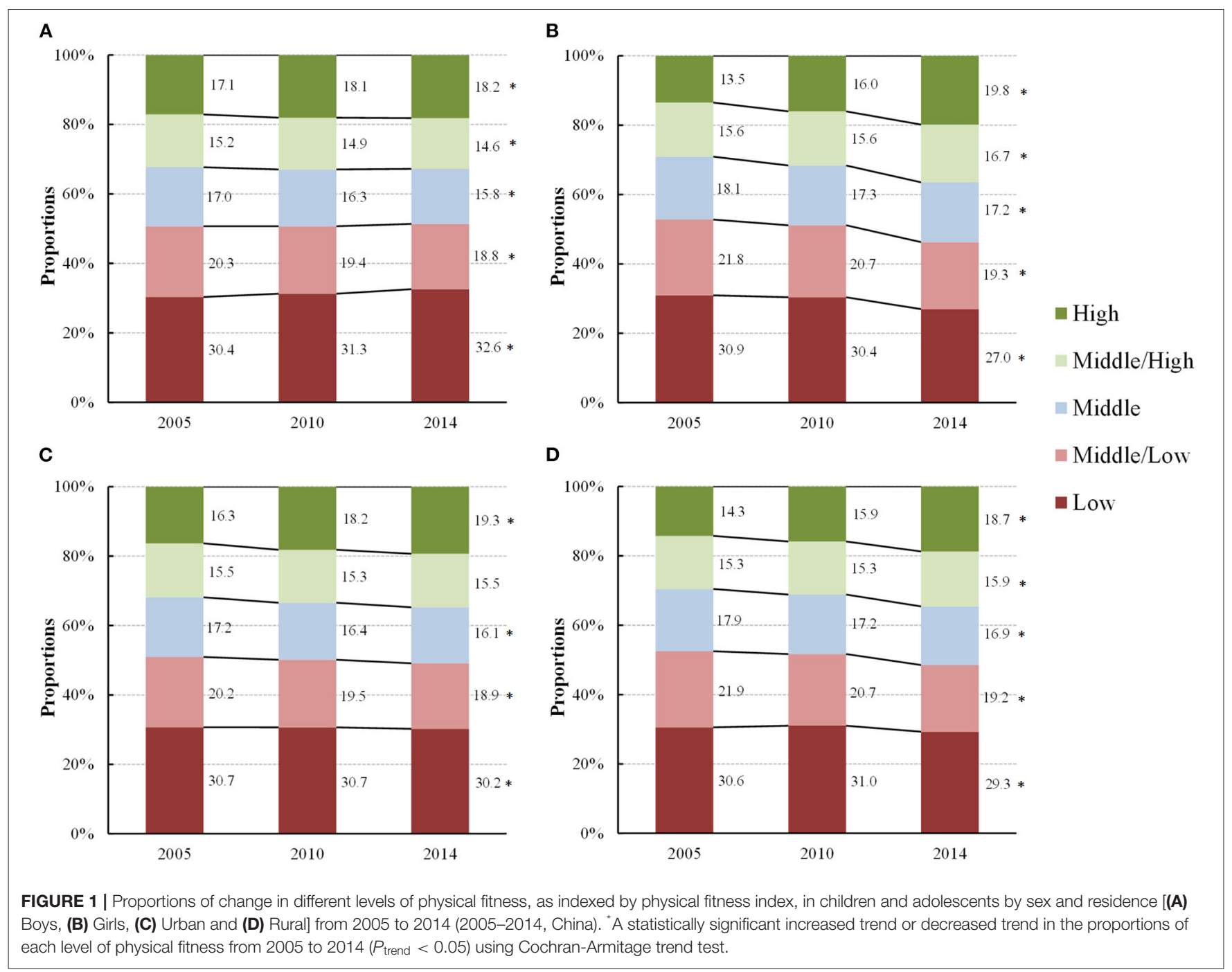

eastern coastal provinces, such as Jiangsu, Zhejiang, Shanghai, Beijing, and Fujian, exhibited the largest proportion of high level of PFI. Up to 2014, the proportions of high level of PFI in the eastern, central, western and northeastern regions were $28.5,16.4,12.5$, and $16.5 \%$, respectively (Table 2 ). Students living in the eastern regions also witnessed a largest increase in the proportion of high level of PFI (6.7 percent point from 2005 to 2014), followed by those living in the western and central regions (2.8 and 2.0 percent point from 2005 to 2014), but those from northeastern regions even showed a decrease in high level of PFI from $17.8 \%$ in 2005 to $16.5 \%$ in 2014 with 1.3 percent point decreases.

On the contrary, children and adolescents in the western and northeastern provinces showed a high proportion of low levels of PFI, followed those living in the central and eastern provinces. The proportions of low levels of PFI in the eastern, central, western and northeastern provinces in 2005 were 21.0, $30.8,38.7$, and $33.2 \%$ respectively. In 2014 , their estimates were changed to 21.6, 28.6, 36.0, and 36.4\%, respectively. Across the four geographic regions, children and adolescents in the western provinces showed the largest decrease (by 2.7 percentage points) in the proportion of low levels of PFI from 2005 to 2014, though there was also an increase, between 2005 and 2014, for those living in the eastern and northeastern provinces. Specifically, children and adolescents in Gansu, Qinghai, and Tibet were shown to have the largest decrease in the proportion of low levels of PFI (by 15.7, 15.4, and $15.1 \%$ points). In contrast, children and adolescents in Guangxi and Ningxia exhibited the largest increase in the proportion of low levels of PFI (by 15.0 and $14.7 \%$ points, respectively (Figure 4 and Supplementary Table 2). The standardized values of each physical fitness components were also showed in the Supplementary Table 3. The FVC, 50 meter dash, and endurance running among children and adolescents got improvement from 2005 to 2014 in four regions, particularly in central and western regions, but the standing long jump, sit and reach 


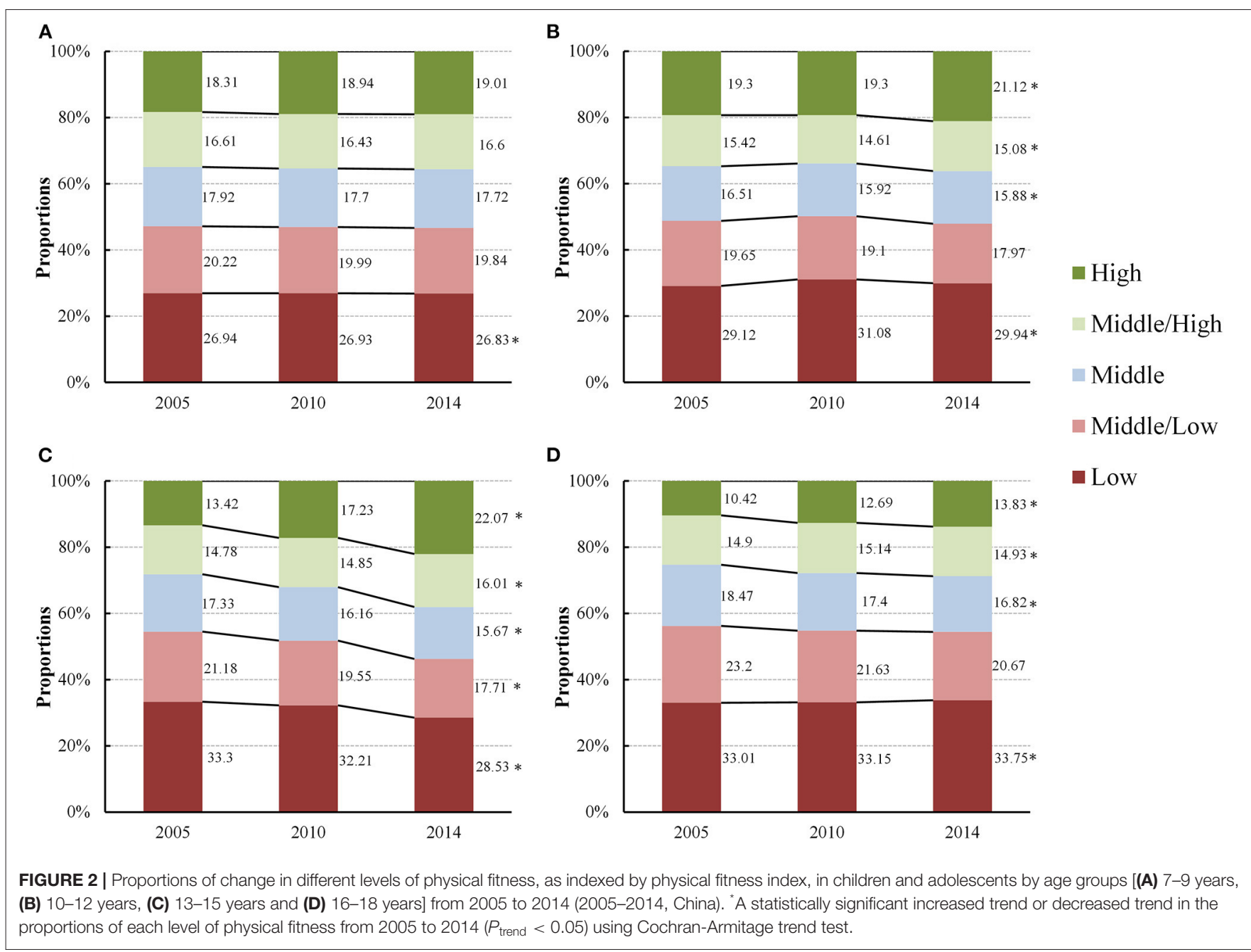

and body muscle strength deteriorated in 2014 than they were in 2005.

\section{DISCUSSION}

To our knowledge, this is the first study that used three successive national cross-sectional surveys between 2005 and 2014 that assessed the geographic variations in comprehensive physical fitness among Chinese school-aged children and adolescents. From 2005 to 2014, there was an increase in the proportion of high PFI and a decrease in the proportion of low PFI among Chinese children and adolescents, especially among girls, and those living in rural areas and in middle-school stage. These trends were observed with notable geographic variability showing that children and adolescents in the eastern provinces having a higher proportion of PFI compared with a higher proportion of low PFI in children and adolescents living in the western provinces. During the study period, the levels of physical fitness among children and adolescents in both eastern and western provinces showed an upward (in the direction of improvement) trend.

Actually, there has been an increase in health-promotion policies at national and local levels. In 2007, the State Council of the Chinese government issued a strategic policy aimed at strengthening and enhancing physical fitness for schoolaged children and adolescents (17). In the years followed, the government issued a total of 88 multisectoral measures and policies involving promotion of sport, reductions in academic burden through curriculum reform, and development of public facilities to promote students' physical fitness $(12,18)$. Although there has been an increase in health-promotion policies in China, our findings highlight notable geographic variations in the levels of children's physical fitness across regions with children living in the eastern provinces experiencing high levels of fitness compared to those living in the western provinces. These geographic disparities may reflect an uneven development and implementation of nationwide policies. In order to promote physical fitness among children and adolescents, China initiated its Healthy China 2030 blueprint in 2016 (19). It set out specific requirements on physical activities for children and puts them 


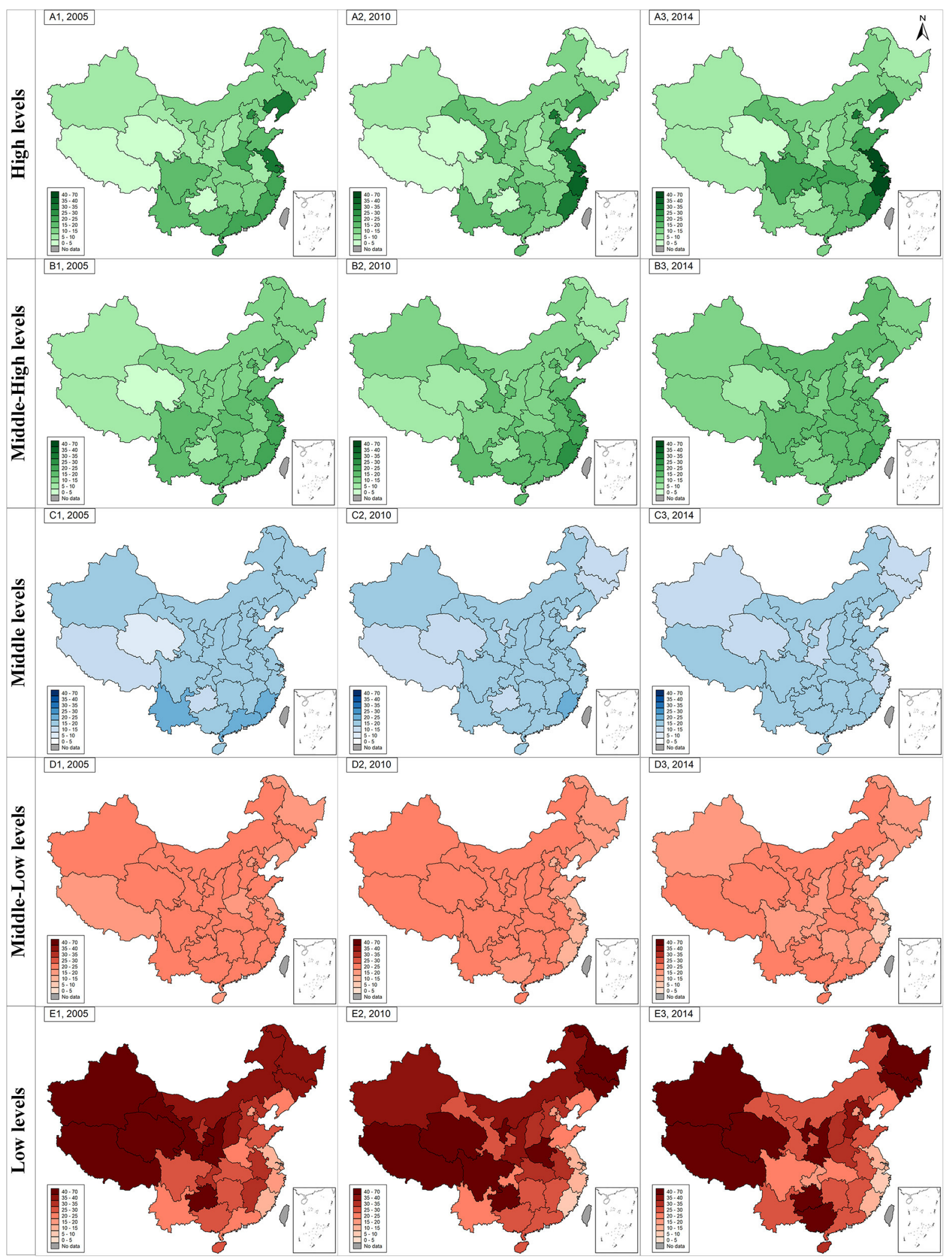

FIGURE 3 | The geographical distribution and changes of different levels of physical fitness index from 2005 to 2014 (2005-2014, China). The green graph in subfigure (A1, A2, and A3 for 2005, 2010, and 2014) and (B1, B2, and B3 for 2005, 2010, and 2014) represents geographical distribution of high levels of PFI and middle-high levels of PFI in 2005, 2010, and 2014. The blue graph in subfigure (C1, C2, and C3 for 2005, 2010, and 2014) represents geographical distribution of middle levels of PFI in 2005, 2010, and 2014. The red graph in subfigure (D1, D2, and D3 for 2005, 2010, and 2014) and (E1, E2, and E3 for 2005, 2010, and 2014) represents geographical distribution of low levels of PFI and middle-low levels of PFI in 2005, 2010, and 2014. The gradient of each color series in each subfigure was used to show the exact proportions of the specific PFI levels (exact number showed in Supplementary Table 2). 
TABLE 2 | The geographical changes of different levels of physical fitness index from 2005 to 2014.

\begin{tabular}{|c|c|c|c|c|}
\hline Categories & $2005, \%$ & $2010, \%$ & $2014, \%$ & $\begin{array}{c}\text { 2005-2014 Changes } \\
\text { (Percent point, } P \\
\text { values) * }\end{array}$ \\
\hline \multicolumn{5}{|l|}{ High Level } \\
\hline East & 21.8 & 27.0 & 28.5 & $6.7(<0.001)$ \\
\hline Central & 13.4 & 12.0 & 16.4 & $3.0(<0.001)$ \\
\hline West & 9.6 & 11.7 & 12.5 & $2.8(<0.001)$ \\
\hline Northeast & 17.8 & 13.7 & 16.5 & $-1.3(0.001)$ \\
\hline \multicolumn{5}{|c|}{ Middle-High level } \\
\hline East & 18.7 & 19.3 & 17.6 & $-1.2(<0.001)$ \\
\hline Central & 15.5 & 14.4 & 16.4 & $0.9(0.001)$ \\
\hline West & 12.7 & 13.1 & 14.0 & $1.3(<0.001)$ \\
\hline Northeast & 14.2 & 11.7 & 14.0 & $-0.1(0.739)$ \\
\hline \multicolumn{5}{|l|}{ Middle Level } \\
\hline East & 18.9 & 17.7 & 16.0 & $-3.0(<0.001)$ \\
\hline Central & 18.2 & 18.1 & 18.1 & $-0.1(0.701)$ \\
\hline West & 16.6 & 16.2 & 16.7 & $0.1(0.634)$ \\
\hline Northeast & 15.5 & 13.5 & 14.8 & $-0.7(0.053)$ \\
\hline \multicolumn{5}{|c|}{ Low-Middle Level } \\
\hline East & 19.6 & 17.2 & 16.3 & $-3.3(<0.001)$ \\
\hline Central & 22.2 & 22.8 & 20.6 & $-1.6(<0.001)$ \\
\hline West & 22.4 & 21.7 & 20.9 & $-1.5(<0.001)$ \\
\hline Northeast & 19.4 & 18.0 & 18.2 & $-1.2(0.002)$ \\
\hline \multicolumn{5}{|l|}{ Low Level } \\
\hline East & 21.0 & 18.8 & 21.6 & $0.7(0.002)$ \\
\hline Central & 30.8 & 32.8 & 28.6 & $-2.2(<0.001)$ \\
\hline West & 38.7 & 37.4 & 36.0 & $-2.7(<0.001)$ \\
\hline Northeast & 33.2 & 43.1 & 36.4 & $3.2(<0.001)$ \\
\hline
\end{tabular}

*The difference of proportions of each level of PFI in different survey years between 2005 and 2014 years was evaluated by the Chi-square test.

into action, which makes school-aged children achieve at least $1 \mathrm{~h}$ of physical activity daily and have more than $25 \%$ of them achieve an "excellent" rating in physical fitness. Thus, the geographic variations in physical fitness in this study will clarify the disadvantaged areas and the priority of public health resources investment under the national Healthy China 2030 strategy.

Findings from this study highlight significant geographic variations in the levels of fitness among children and adolescents living between economically developed regions (e.g., eastern provinces) and those that are less well-developed (e.g., western provinces). It is conceivable that such variation may reflect geographic disparities in economic development, investment in physical activity or sport facilities, and inherent uneven resources. As such, in order to narrow these disparities, targeted physical fitness interventions or programs should be directed toward school children living in those under developed areas (e.g., western provinces). From the health promotion perspective, priority areas in physical fitness programs need to be identified across regions of a wide socioeconomic spectrum and be targeted at the province, district, school and classroom levels, through policy and practice recommendations, periodical physical fitness and health surveillance, and implementation of evidence-based health-related school PE curricula (2022).

Apart from those nationwide efforts, reductions in academic pressures, breaking of sedentary time, and engagement in sporting activities should also be encouraged to narrow these regional disparities in children and adolescents' physical fitness (23). For example, since obesity and undernutrition were both important causes of physical fitness deterioration, the background of rapid increase in obesity and persistent undernutrition among children and adolescents suggested that the differentiated improvement measures were needed in different regions of China $(24,25)$. Thus, as suggested in the previous study, the control of over-nutrition should be emphasized among eastern developed provinces with policies that encourage taxation on unhealthy foods and promotion of moderate-to-vigorous PA. In the less developed western provinces, emphasis should be placed on improving malnutrition with promotion of rural nutrition improvement programs and dietary diversity (12).

Our study has several strengths, derived from its comprehensive assessment of physical fitness combining respiratory function, strength, flexibility, explosive power, and 


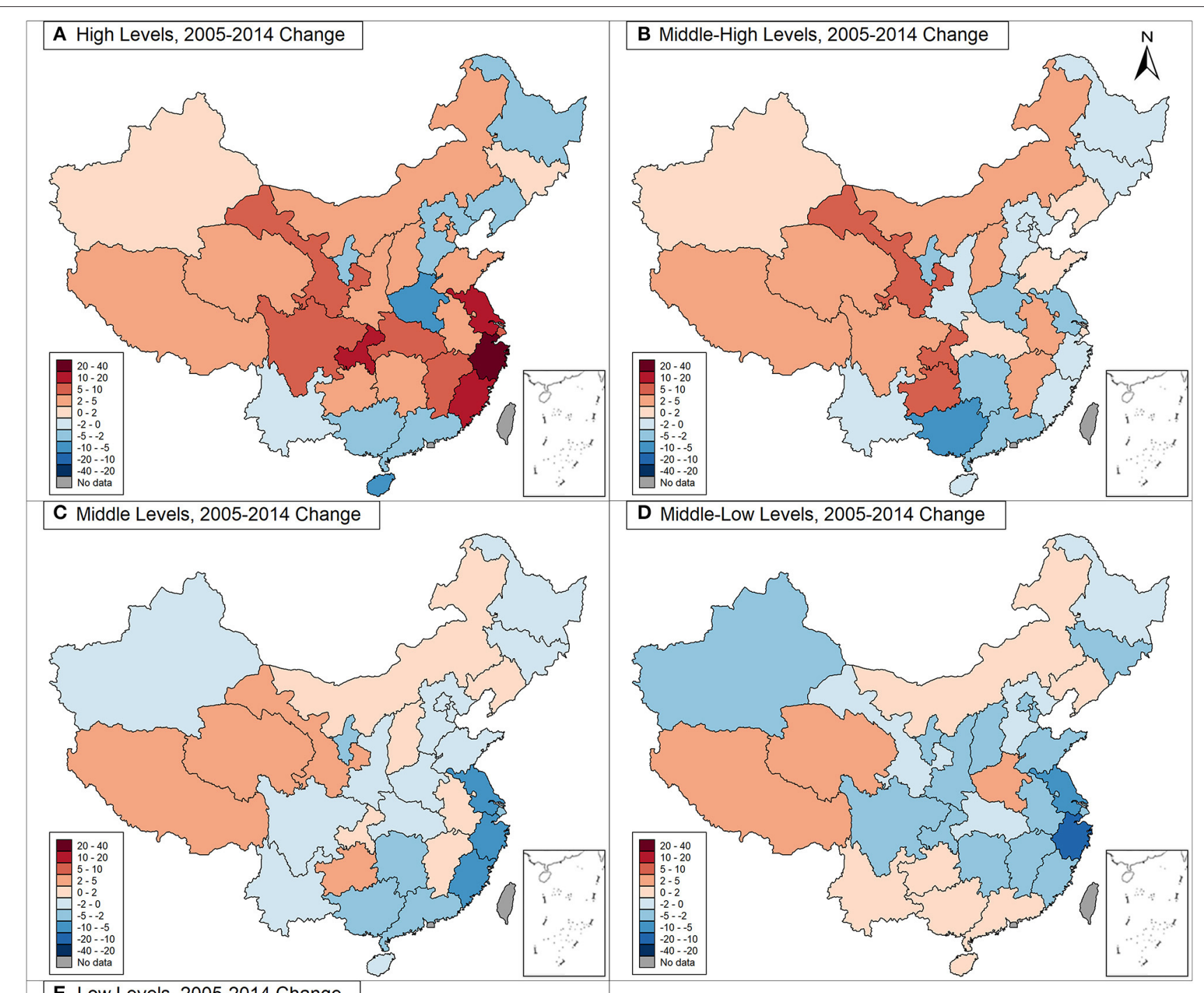

\section{E Low Levels, 2005-2014 Change}

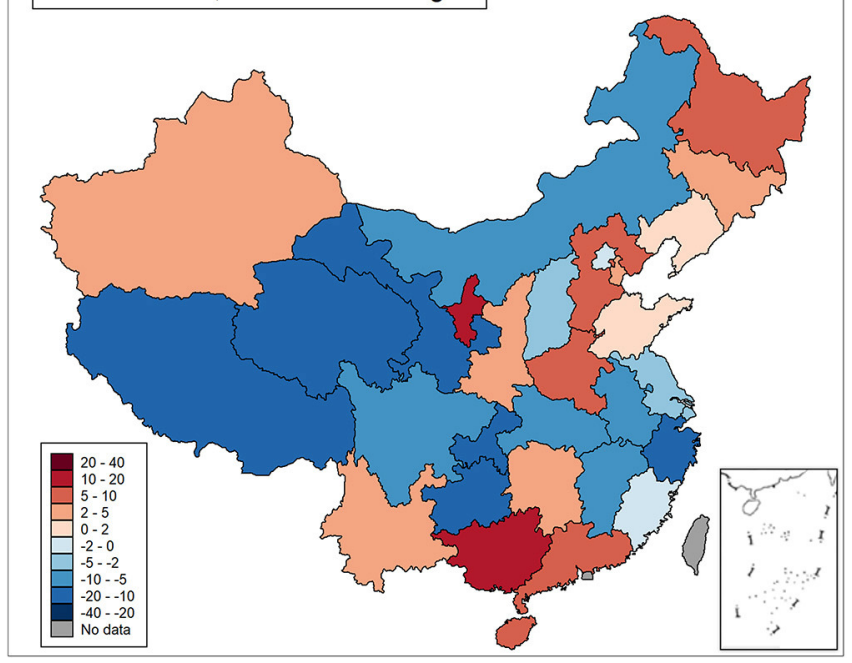

FIGURE 4 | The changes of different levels of physical fitness from 2005 to 2014 (2005-2014, China). (A) For high levels of PFI; (B) for middle-high levels of PFl; (C) for middle levels of PFI; (D) for middle-low levels PFI; (E) for low levels PFI. The red-colored item in subfigure represents an increased percentage point of each level physical fitness index from 2005 to 2014. The blue-colored item in subfigure represents a decreased percentage point of each level physical fitness index from 2005 to 2014. The color gradient of red and blue series was used to show the proportions' changes of each level of PFI (exact number showed in Supplementary Table 2). 
cardiorespiratory endurance factions, with a large nationallyrepresentative sample size and repeated measures with geographic variations across a 10-year period. Our study also has several limitations that should be noted. First, since this study used original data on subnational changes from three cross-sectional surveys, the data cannot be used to infer causality, and merely ecological analyses were adopted in this study. However, the CNSSCH collected nationally representative data with a large sample size and compelling evidence, and the comprehensive physical fitness estimated in CNSSCH could meet our purpose of secular trend, geographical and influencing factors analyses. Second, our study adopted the PFI assessing the comprehensive physical fitness, and did not use the national fitness standard, and also some characteristics of six components of PFI might be ignored. The scope of PFI could be not as broad as the national fitness standard in the use of the individual evaluation of physical fitness. But it has other advantages, for example, it was helpful to observe the different levels of physical fitness, including the low, low-middle, middle, middle-high, and high levels of PFI analyzed in this study, and extend the application scope in the use of PFI in terms of trend analysis and intra-data comparison. In addition, the national fitness standards were calculated based on grade rather than age, which did not apply to our data.

\section{CONCLUSIONS}

Findings from this study showed an improved trend, from 2005 to 2014, in the overall level of physical fitness among Chinese children and adolescents. However, notable geographic variations in the levels of fitness were observed with high prevalence of healthy fit children and adolescents living in the eastern region and unhealthy or low-fit children and adolescents living in the western region of the country. These findings suggest that national and regional school fitness healthenhancing strategies and initiatives should aim to reduce geographic disparities by targeting evidence-based interventions in geographical regions that have a high prevalence of unhealthy physical fitness in school children and adolescents across the country from diverse regions.

\section{DATA AVAILABILITY STATEMENT}

All the individual (de-identified) participant data collected in the surveys can be shared with investigators whose proposed use of the data has been approved by an independent review committee identified for this purpose by contacting the corresponding

\section{REFERENCES}

1. Malina RM. Physical fitness of children and adolescents in the United States: status and secular change. Med Sport Sci. (2007) 50:67-90. doi: 10.1159/000101076

2. Huang YC, Malina RM. BMI and health-related physical fitness in Taiwanese youth 9-18 years. Med Sci Sports Exerc. (2007) 39:7018. doi: $10.1249 / \mathrm{mss} .0 \mathrm{~b} 013 \mathrm{e} 31802 \mathrm{f} 0512$ author. Proposals should be directed to majunt@bjmu.edu.cn and songyi@bjmu.edu.cn.

\section{ETHICS STATEMENT}

The studies involving human participants were reviewed and approved by the Medical Research Ethics Committee of the Peking University Health Science Center. Written informed consent to participate in this study was provided by the participants' legal guardian/next of kin.

\section{AUTHOR CONTRIBUTIONS}

YD conceptualized and designed the study, completed the statistical analyses, drafted the initial manuscript, and reviewed and revised the manuscript. JM and YS contributed to the conceptualization and design of the study, supervised the data collection, the statistical analyses and initial drafting of the manuscript, and reviewed and revised the manuscript. PL participated in conceiving the study design and critically reviewed and revised the manuscript from preliminary draft to submission. MC assisted with the data interpretation, and reviewed and revised the manuscript. All authors approved the final manuscript as submitted and agreed to be accountable for all aspects of the work.

\section{FUNDING}

The present study was supported by the National Natural Science Foundation (Grant 81673192 to JM), and Humanities and Social Sciences Planning Fund Project, Ministry of Education, People's Republic of China (19YJA890022 to YS), as well as project funded by the China Postdoctoral Science Foundation (BX20200019 and 2020M680266 to YD).

\section{ACKNOWLEDGMENTS}

The authors would like to acknowledge the support from all the team members and the participating students, teachers, parents, and local education and health staff in the programs.

\section{SUPPLEMENTARY MATERIAL}

The Supplementary Material for this article can be found online at: https://www.frontiersin.org/articles/10.3389/fpubh. 2021.694070/full\#supplementary-material 
Prev Rehabil. (2009) 16:677-83. doi: 10.1097/HJR.0b013e32833 $12 \mathrm{e} 94$

5. Fagard RH. Physical activity, physical fitness and the incidence of hypertension. $J$ Hypertens. (2005) 23:2657. doi: 10.1097/00004872-200502000-00005

6. Carnethon MR, Evans NS, Church TS, Lewis CE, Schreiner PJ, Jacobs JR, et al. Joint associations of physical activity and aerobic fitness on the development of incident hypertension: coronary artery risk development in young adults. Hypertension. (2010) 56:49-55. doi: 10.1161/HYPERTENSIONAHA.109.147603

7. Blair SN, Cheng Y, Holder JS. Is physical activity or physical fitness more important in defining health benefits? Med Sci Sports Exerc. (2001) 33:S37999. doi: 10.1097/00005768-200106001-00007

8. Malina RM, Katzmarzyk PT. Physical activity and fitness in an international growth standard for preadolescent and adolescent children. Food Nutr Bull. (2006) 27:S295-313. doi: 10.1177/15648265060274S511

9. Macfarlane DJ, Tomkinson GR. Evolution and variability in fitness test performance of Asian children and adolescents. Med Sport Sci. (2007) 50:14367. doi: 10.1159/000101358

10. Tomkinson GR, Macfarlane D, Noi S, Kim DY, Wang Z, Hong R. Temporal changes in long-distance running performance of Asian children between 1964 and 2009. Sports Med. (2012) 42:267-79. doi: 10.2165/11599160-000000000-00000

11. Tomkinson GR, Olds TS. Pediatric fitness: secular trends and geographic variability. J Sports Sci Med. (2007) 6:267.

12. Dong Y, Lau PWC, Dong B, Zou Z, Yang Y, Wen B, et al. Trends in physical fitness, growth, and nutritional status of Chinese children and adolescents: a retrospective analysis of 1.5 million students from six successive national surveys between 1985 and 2014. Lancet Child Adolesc Health. (2019) 3:87180. doi: 10.1016/S2352-4642(19)30302-5

13. Dong Y, Jan C, Ma Y, Dong B, Zou Z, Yang Y, et al. Economic development and the nutritional status of Chinese school-aged children and adolescents from 1995 to 2014: an analysis of five successive national surveys. Lancet Diabetes Endocrinol. (2019) 7:288-99. doi: 10.1016/S2213-8587(19)30075-0

14. Dong Y, Ma J, Song Y, Dong B, Wang Z, Yang Z, et al. National Blood Pressure Reference for Chinese Han Children and Adolescents Aged 7 to 17 Years. Hypertension. (2017) 70:897-906. doi: 10.1161/HYPERTENSIONAHA.117.09983

15. Dong Y, Ma J, Song Y, Ma Y, Dong B, Zou Z, et al. Secular Trends in Blood Pressure and Overweight and Obesity in Chinese Boys and Girls Aged 7 to 17 Years From 1995 to 2014. Hypertension. (2018) 72:298305. doi: 10.1161/HYPERTENSIONAHA.118.11291

16. National Bureau of Statistics of China (2012). East-west and east-northeast division methods in China. Available online at: http://www.stats.gov.cn/ztjc/ zthd/sitjr/dejtjkfr/tjkp/201106/t20110613_71947.htm (accessed June 2011).

17. Notification on strengthening sports and enhancing the physical fitness of children and youth from China central committee and the state council. Available online at: http://www.moe.gov.cn/jyb_xxgk/moe_1777/moe_1778/ tnull_27692.html2007.
18. Song Y, Luo DM, Hu PJ, Yan XJ, Zhang JS, Lei YT, et al. [Trends of prevalence of excellent health status and physical fitness among Chinese Han students aged 13 to 18 years from 1985 to 2014]. Beijing Da Xue Xue Bao Yi Xue Ban. (2020) 52:317-22. doi: 10.19723/j.issn.1671-167X.2020.02.020

19. State Council of China. Available online at: Healthy China 2030 Blueprint Guide. http://www.gov.cn/zhengce/2016-10/25/content_5124174.htm (accessed Feb 28, 2021).

20. Lee SA, Ju YJ, Lee JE, Hyun IS, Nam JY, Han KT, et al. The relationship between sports facility accessibility and physical activity among Korean adults. $B M C$ Public Health. (2016) 16:893. doi: 10.1186/s12889-016-3574-z

21. Bull FC, Al-Ansari SS, Biddle S, Borodulin K, Buman MP, Cardon G, et al. World Health Organization 2020 guidelines on physical activity and sedentary behaviour. Br J Sports Med. (2020) 54:1451-62. doi: 10.1136/bjsports-2020-102955

22. Heath GW, Parra DC, Sarmiento OL, Andersen LB, Owen N, Goenka S, et al. Evidence-based intervention in physical activity: lessons from around the world. Lancet. (2012) 380:272-81. doi: 10.1016/S0140-6736(12)60816-2

23. Chaput JP, Willumsen J, Bull F, Chou R, Ekelund U, Firth J, et al. 2020 WHO guidelines on physical activity and sedentary behaviour for children and adolescents aged 5-17 years: summary of the evidence. Int J Behav Nutr Phys Act. (2020) 17:141. doi: 10.1186/s12966-020-01037-Z

24. Dong Y, Ma Y, Dong B, Zou Z, Hu P, Wang Z, et al. Geographical variation and urban-rural disparity of overweight and obesity in Chinese school-aged children between 2010 and 2014: two successive national crosssectional surveys. BMJ Open. (2019) 9:e025559. doi: 10.1136/bmjopen-2018025559

25. Dong Y, Bennett K, Jan C, Dong B, Zou Z, Hu P, et al. Subnational variation of stunting, wasting and malnutrition in Chinese primary-school children between 2010 and 2014: urban-rural disparity. Public Health Nutr. (2019) 1-12. doi: $10.1017 /$ S1368980019000235

Conflict of Interest: The authors declare that the research was conducted in the absence of any commercial or financial relationships that could be construed as a potential conflict of interest.

Publisher's Note: All claims expressed in this article are solely those of the authors and do not necessarily represent those of their affiliated organizations, or those of the publisher, the editors and the reviewers. Any product that may be evaluated in this article, or claim that may be made by its manufacturer, is not guaranteed or endorsed by the publisher.

Copyright $\odot 2021$ Dong, Chen, Song, Ma and Lau. This is an open-access article distributed under the terms of the Creative Commons Attribution License (CC BY). The use, distribution or reproduction in other forums is permitted, provided the original author(s) and the copyright owner(s) are credited and that the original publication in this journal is cited, in accordance with accepted academic practice. No use, distribution or reproduction is permitted which does not comply with these terms. 